\title{
A comparison of two algorithms for solving closed crack problems
}

\author{
Jean-Claude De Bremaecker ${ }^{\mathrm{a} *}$, Michael Ferris ${ }^{\mathrm{b}}$
}

${ }^{\mathrm{a}}$ Department of Geology and Geophysics, Rice University, Box 1892, Houston, TX 77251

${ }^{\mathrm{b}}$ Department of Computer Sciences, University of Wisconsin, Madison, WI 53706

In a recent paper Elvin and Leung [1] demonstrated the advantages of a new fast boundary element algorithm for solving closed crack problems. This algorithm uses the Displacement Discontinuity Method (DDM) and subdivides the problem into two sub-problems, one for the outer boundaries of the structure and one for the crack. In common with most methods for crack problems, it uses interface -- or joint -- elements to prevent interpenetration, following the scheme described by Crouch [2] (pp. 208-212). As we shall see, the use of such elements introduces errors of unknown magnitude in the computation of the stress intensity factor, whether the numerical scheme is the DDM, as in the present case, or the finite element method as in [3]. These errors appear not to have been noticed or explained. Thanks to the courtesy of Dr. Elvin, we have been able to compare the results obtained by [1] for their second problem with those obtained with the PATH algorithm [4-6] applied to a complementary formulation of the problem [7]. We will show that this formulation combined with the PATH algorithm eliminates these errors, albeit at the expense of some increase in computer time. We wish to thank Dr. Elvin both for giving us the details of their model and for many fruitful discussions.

In the problem here examined a shallow crack EF (see Fig. 1) is subjected to compressive loading from a succession of surface point loads applied between B and C. (Details of the model are given in Table 1.) One wishes to determine the value of the stress intensity factor at one or both crack tips, the parts of the crack which are open, those which are in contact and slipping, and those which are stuck. It is important to note that the effect of each load is considered independently of the effects of the others (Elvin, pers. comm., 1999), see also [3].

In order to make the comparison of the two algorithms as meaningful as possible, we used exactly the same model as [1] with the following exceptions:

-- in [1] the elastic constants of the joint elements are $K_{S}=K_{N}=2 \times 10^{17}$ and Young's modulus $\mathrm{E}=10^{11}$; there are no joint elements in our model and $\mathrm{E}=1.0$;

-- [1] uses quadratic displacement discontinuity elements (QDD) at the surface and constant displacement discontinuity elements (CDD) on the crack (Elvin, pers. com., 1999), while we use CDD everywhere because our code does not support QDD at present. In order approximately to compensate for this difference we use three times as many elements on the surface, and thus obtain the same number of nodes as [1]. 
-- [1] used an INDY Silicon Graphics with a $150 \mathrm{MHz}$ processor. We used a Sun Ultrasparc Ili with a $269 \mathrm{MHz}$ processor. As a rough approximation this results in 1.8 higher speed.

The results obtained by [1] are shown in Figure 2, but in figure $2 \mathrm{a}$ (top) the $\mathrm{x}$ - and $\mathrm{y}$-axes (or $x_{p} / a$ and $\mathrm{x} / \mathrm{a}$ ) have been interchanged from those in figure 12 of [1] to allow an easier correlation with figure $2 \mathrm{~b}$ (bottom). Figure $2 \mathrm{a}$ shows the open, slip, and stuck regions of the crack as the load at the surface moves from point B to point C. (In the figure itself the words "Right" and "Left" refer to the fact that an observer on either side of the crack sees the other side moving to the right or left, respectively; the letter $\mathrm{S}$ designates the stuck region.). In this figure the situation at the left crack tip is that shown on the $x_{\mathrm{p}} / \mathrm{a}$-axis, for which $\mathrm{x} / \mathrm{a}=-1$.

Figure $2 b$ shows the corresponding variation of the normalized Stress Intensity Factor $\left(\mathrm{SIF}^{\mathrm{N}}\right.$ $=\mathrm{K}_{\mathrm{II}}^{\mathrm{N}}$ ) at the left crack tip, both from [1] (Fig. 11; the actual data were given to us by Dr. Elvin), shown by open circles, and from our own, shown by asterisks. It should however be noted that the ordinates of our curve have been divided by 1.16; considering that the models are essentially identical, and use identical elements on the crack, this $16 \%$ difference in the values of $\mathrm{SIF}^{\mathrm{N}}$ must be ascribed to the presence of joint elements in [1] and their absence in our method.

As the point of application of the load moves from $\mathrm{x}_{\mathrm{p}} / \mathrm{a} \approx-2.0$ (point $\mathrm{B}$ ) towards the right, $\mathrm{SIF}^{\mathrm{N}}$ first increases, and passes through a maximum at $\mathrm{x}_{\mathrm{p}} / \mathrm{a} \approx-1.5$, which is exactly above the crack tip. As the load moves further to the right, the crack tip approaches the stuck region, correspondingly $\mathrm{SIF}^{\mathrm{N}}$ decreases, as is seen both in the results of [1] and our own, although in ours the decrease is much more rapid. The difference between the results becomes very pronounced for $\mathrm{x}_{\mathrm{p}} / \mathrm{a} \approx-1.2$; figure $2 \mathrm{a}$ shows that this is where the crack tip becomes stuck. In our results the $\mathrm{SIF}^{\mathrm{N}}$ becomes null at this point, and, as expected, stays null throughout the stuck (S) region, i.e., until $x_{p} / a \approx-0.7$. This is not the case in the results of [1]. The cause for this phenomenon is not immediately obvious, since $\mathrm{SIF}^{\mathrm{N}}=\mathrm{K}_{\mathrm{II}}{ }^{\mathrm{N}}=\mathrm{A} \times \mathrm{ds}_{\text {tip }}$ where $\mathrm{A}$ is a proportionality factor which need not concern us here.

The reason for this seeming contradiction is that two different definitions of the stuck region are possible, i.e., in the first one it corresponds to $\mathrm{ds}_{\mathrm{tip}}=0$, in the other one to $\tau<\sigma \times \mathrm{f}$ ( $\tau$ is the shear stress, $\sigma$ is the normal stress, and $\mathrm{f}$ is the friction coefficient). These two definitions are identical if the friction law is strictly observed, but the presence of joint elements may cause them to differ slightly, so that it is possible to have $\mathrm{ds}_{\text {tip }}$ ? 0 and $\tau<\sigma \times \mathrm{f}$, or $\mathrm{ds}_{\text {tip }}=0$ and $\tau=\sigma \times \mathrm{f}$ at the same point, in contradiction to the friction law. A very similar phenomenon may be seen in [3], and we thank Professor Komvopoulos for his discussion of the problem. 
Our results for the open, slip and stuck regions differ very little from those obtained by [1], and warrant no further discussion.

We now turn to the time required for the solution, remembering that the number of nodes is the same in both cases. The fast algorithm of [1] takes approximately $15 \mathrm{~s}$, while his conventional, or direct method takes roughly 225s (Elvin, pers. comm.). The PATH algorithm itself takes approximately $11 \mathrm{~s}$ on our faster machine, corresponding roughly to $20 \mathrm{~s}$ on the machine used in [1]; this time varies slightly depending on the "difficulty" of the problem; the PATH algorithm is written in C. The complete solution of the problem takes about 26 to $32 \mathrm{~s}$; the main reason for this increase in computer time is that the program itself is written in the GAMS language [8], which is interpretive and thus slower.

This increase in computer time should not be underestimated; on the other hand the error due to the use of joint elements in the computation of SIF $^{\mathrm{N}}$ appears to be of the order of $16 \%$ as shown above, while the difference in the shape of the two curves of SIF $^{\mathrm{N}}$ is also large. It should further be pointed out that the PATH algorithm computes and displays the maximum error between the solution obtained and the friction law; it is $=1 \times 10^{-9}$ in the cases shown here, for which $\mathrm{ds}_{\text {tip }}$ varies between 0.0 and $1.5 \times 10^{-3}$. Finally, it should be noted that the present case involves only the simplest possible geometrical configuration; even in this case the difference between the results obtained using joint elements and those obtained with the PATH algorithm is far from negligible. If the geometry were more complex, e.g., multiple cracks or cracks with sharp changes in direction, the advantage of using an algorithm of known accuracy might be appreciable and more than compensate the increase in computation time.

It may be of some interest to note that we have also examined the following cases:

-- if the load is applied over a segment $4 \times 10^{-4}$ rather than 0.1 as above, the only important difference is the much greater extent of the open regions, as might be intuitively expected.;

-- if the number of elements on the crack is multiplied by a factor of 3 , the value of the $\operatorname{SIF}^{\mathrm{N}}$ is further increased by a factor of about 1.08; such an increase is expected and is due to the fact that $\mathrm{ds}_{\text {tip }}$ is now evaluated closer to the crack tip;

-- finally, if the crack is embedded in a finite body of 100 'depth', the results are again essentially unchanged, also as expected.

\section{Acknowledgment}

Computer time was made available by the Department of Geology and Geophysics, Rice University. 


\section{References}

1. Elvin N, Leung C. A fast iterative boundary element method for solving closed crack problems, Engineering Fracture Mechanics 1999; 63: 631-648.

2. Crouch SL, Starfield AM, Boundary Element Methods in Solid Mechanics, 1983 (reprinted, 1990), George Allen and Unwin, $322 \mathrm{pp.}$

3. Komvopoulos K. Subsurface crack mechanisms under indentation loading, Wear 1995; 199: 9-23.

4. Dirkse SP, Ferris MC. The PATH solver: A non-monotone stabilization scheme for mixed complementarity problems, Optimization Methods and Software 1995; 5: 123-156.

5. Ferris MC, Munson TS. Complementarity problems in GAMS and the PATH solver, J. of Economic Dynamics and Control 2000; 24: 165-188.

6. Ralph D. Global convergence of damped Newton's method for nonsmooth equations via the Path search, Mathematics of Operations Research 1994; 19: 352-389.

7. De Bremaecker J-C, Ferris MC,Ralph D. Compressional fractures considered as contact problems and mixed complementarity problems, subm.; .

8. Brooke A, Kendrick D, Meeraus A,Raman R, GAMS, A user's guide, 1998, GAMS Development Corp., Washington, DC, 262 pp. 


\section{Figures}

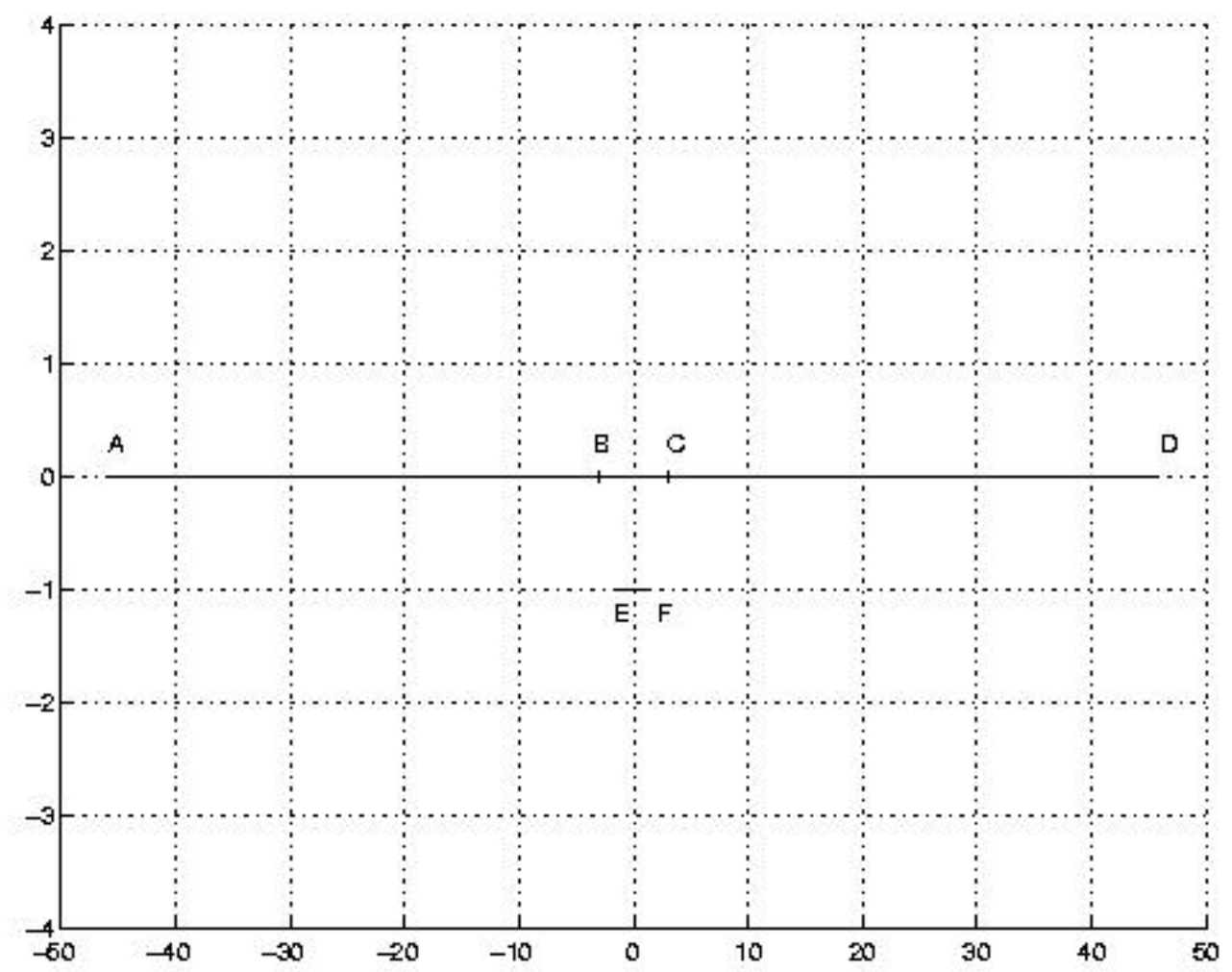

Figure 1: The part of the free surface considered consists of three segments, $A B, B C$, and $\mathrm{CD}$; the embedded crack is segment EF. See Table 1 for details. Note the difference in horizontal and vertical scales. 
EFM-Res. note-FMT 1/25/0025-Jan-00
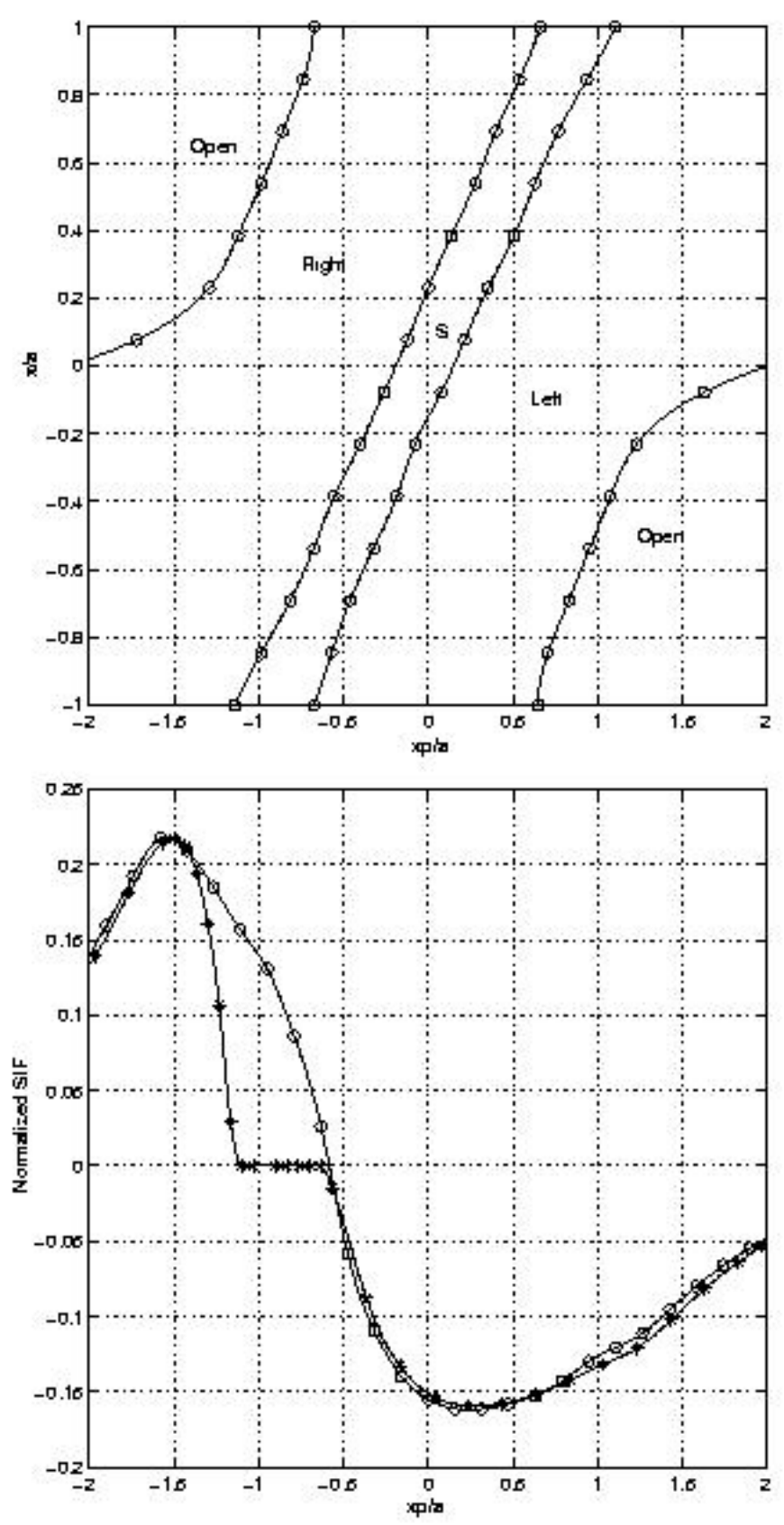

6

Figure 2

a. Open, right slipping (Right), stuck (S), and left slipping (Left) parts of the crack plotted vs. the normalized position of the point load at the surface. $\mathrm{x}_{\mathrm{p}}$ : coordinate of the point load, a: half-length of the crack; $x$ : coordinate of a point on the crack. Reproduced from [1] (Fig. 12) but with an interchange of the $\mathrm{x}$ - and $\mathrm{y}$-axes.

b. Normalized Stress Intensity Factor, $\mathrm{SIF}^{\mathrm{N}}=\mathrm{K}_{\mathrm{II}}{ }^{\mathrm{N}}=\mathrm{K}_{\mathrm{II}} \times \pi \mathrm{vh} /(2 \mathrm{P}) ; \mathrm{h}=-1.0$ : depth of the crack, P: surface load. 
Table 1: Parameters of the model in [1]

Segment AB, from $(-45.86,0)$ to $(-3,0), 20$ QDD elements, decreasing in length by a factor of 0.8 , length of element closest to B: $0.125 ; \mathrm{BC}: \sigma=\tau=0$.

Segment BC, from $(-3,0)$ to $(3,0), 60$ QDD elements all of length $0.1 ; B C: \sigma=\tau=0$ except at the load element where $\sigma=10, \tau=0$.

Segment CD: mirror image of segment AB.

Segment EF, from $(-1.5,-1)$ to $(1.5,1), 25$ CDD elements all of length 0.12 , crack.

Poisson's ratio $=0.30 ;$ Young's modulus $E=10^{11}$; springs: $\mathrm{K}_{\mathrm{S}}=\mathrm{K}_{\mathrm{N}}=2 \times 10^{17}$. 